\title{
Addition of Amines to Nitriles Catalyzed by Ytterbium Amides: An Efficient One-Step Synthesis of N-aryl Monosubstituted
} Amidines

\author{
Junfeng Wang ${ }^{1}$, Fan $\mathrm{Xu}^{1}$, Tao Cai ${ }^{1}$, and Qi Shen ${ }^{1,2}$ * \\ ${ }^{1}$ Key Laboratory of Organic Synthesis of Jiangsu Province, Department of Chemistry and Chemical \\ Engineering, Dushu Lake Campus, Suzhou University, Suzhou 215123, People's Republic of China. \\ ${ }^{2}$ State Key Laboratory of Organometallic Chemistry, Shanghai Institute of Organic Chemistry, \\ Chinese Academy of Sciences, Shanghai 200032, China
}

qshen@suda.edu.cn

\section{Supporting material}

All manipulations and reactions were performed under a purified argon atmosphere using standard Schlenk techniques. Solvents were degassed and distilled from sodium benzophenone ketyl under argon prior to use. $\mathrm{LYb}(\mathrm{NHAr})(\mathrm{DME})\left(\mathrm{Ar}=2,6-\mathrm{Me}_{2} \mathrm{C}_{6} \mathrm{H}_{3} \mathbf{I}\right.$; 2,6- $-{ }^{\mathrm{i}} \mathrm{Pr}_{2} \mathrm{C}_{6} \mathrm{H}_{3} \quad$ II $), \quad(\mathrm{TMS})_{2} \mathrm{NYb}(\mathrm{L})_{2} \mathrm{YbN}(\mathrm{TMS})_{2} \quad$ III $\quad(\mathrm{L} \quad=$ $\left.\left[\mathrm{Me}_{3} \mathrm{SiNC}(\mathrm{Ph}) \mathrm{N}\left(\mathrm{CH}_{2}\right)_{3} \mathrm{NC}(\mathrm{Ph}) \mathrm{NSiMe}_{3}\right]\right)$ and $\left[(\mathrm{TMS})_{2} \mathrm{~N}\right]_{3} \mathrm{Yb}(\mu-\mathrm{Cl}) \mathrm{Li}(\mathrm{THF})_{3} \quad$ IV were prepared according to the literature procedure. ${ }^{1,2}$ All nitriles and amines were pre-dried, sublimed, recrystallized or redistilled before use. The IR spectra were recorded on a Magna-IR 550 spectrometer. Melting points were determined in sealed Ar-filled capillary tube, and uncorrected. ${ }^{1} \mathrm{H}$ and ${ }^{13} \mathrm{C}$ NMR spectra were recorded on a $300 \mathrm{MHz}$ or $400 \mathrm{MHz}$ 
spectrometer. Chemical shifts $(\delta)$ were reported in ppm. HRMS were recorded on a GCT-TOF instrument.

\section{Procedures:}

General procedure 1: for the direct synthesis of amidines from reaction of amines with nitriles catalyzed by amide I (product 3aa as an Example). A $30 \mathrm{~mL}$ of Schlenk tube under dried argon was charged with the ytterbium amide I ( $82 \mathrm{mg}, 0.095 \mathrm{mmol})$. To the flask were added the benzonitrile $(0.20 \mathrm{~g}, 1.90 \mathrm{mmol}), 2$,6-diisopropylaniline $(0.73 \mathrm{~g}, 3.80$ mmol). The resulting mixture was stirred at $100{ }^{\circ} \mathrm{C}$ for $24 \mathrm{~h}$. The product was isolated by distilling the reaction mixture under vacuum to remove unreacted starting materials. The residue was recrystallized from toluene/hexane to give compound 3aa in $88 \%$ yield.

General procedure 2: for the direct synthesis of triazine from nitriles catalyzed by amide IV (4a as an Example). A $30 \mathrm{~mL}$ of Schlenk tube under dried argon was charged with the ytterbium amide IV $(87 \mathrm{mg}, 0.095 \mathrm{mmol})$. To the flask were added the benzonitrile $(0.20 \mathrm{~g}, 1.90 \mathrm{mmol})$, hexyl amine $(0.38 \mathrm{~g}, 3.80 \mathrm{mmol})$. The resulting mixture was stirred at $100{ }^{\circ} \mathrm{C}$ for $24 \mathrm{~h}$. The product was isolated by distilling the reaction mixture under vacuum to remove unreacted starting materials. The residue was washed with toluene three times to give compound $\mathbf{4 a}$ in $35 \%$ yield.

\section{Analytical data:}

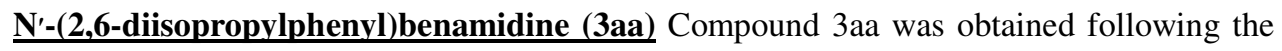


procedure 1 catalyzed by catalysts I-IV and isolated as a white solid in 88, 90, 55 and $45 \%$ yields, respectively.

Mp: $162-163{ }^{\circ} \mathrm{C}$.

$\operatorname{IR}\left(\mathrm{cm}^{-1}\right) v=3433(\mathrm{~N}-\mathrm{H}), 1629(\mathrm{C}=\mathrm{N})$.

${ }^{1} \mathrm{H}$ NMR $\left(\mathrm{CDCl}_{3}\right): \delta=7.92-7.94(2 \mathrm{H}), 7.48-7.50(3 \mathrm{H}), 7.18-7.20(2 \mathrm{H}), 7.08-7.12(1 \mathrm{H})$, $4.62(2 \mathrm{H}), 3.04-3.10(2 \mathrm{H}), 1.21(12 \mathrm{H})$.

${ }^{13} \mathrm{C} \mathrm{NMR}\left(\mathrm{CDCl}_{3}\right): \delta=155.73,144.05,139.95,137.15,129.56,129.37,127.69,123.86$, $123.74,43.65,28.44,24.21,24.08$.

HRMS (ESI): m/z calcd for $\mathrm{C}_{19} \mathrm{H}_{24} \mathrm{~N}_{2}: 280.1939$, found: 280.1931.

4-cloro-N'-(2,6-diisopropylphenyl)benamidine (3ba) Compound 3ba was obtained following the procedure 1 catalyzed by amides I-IV and isolated as white solid in 83,84 , 70 and $20 \%$ yields, respectively.

Mp: $149-150{ }^{\circ} \mathrm{C}$.

$\operatorname{IR}\left(\mathrm{cm}^{-1}\right) v=3431(\mathrm{~N}-\mathrm{H}), 1631(\mathrm{C}=\mathrm{N})$.

${ }^{1} \mathrm{H}$ NMR $\left(\mathrm{CDCl}_{3}\right): \delta=7.84(2 \mathrm{H}), 7.42(2 \mathrm{H}), 7.10-7.15(3 \mathrm{H}), 4.59(2 \mathrm{H}), 2.98(2 \mathrm{H}), 1.18$ $(12 \mathrm{H})$.

${ }^{13} \mathrm{C} \mathrm{NMR}\left(\mathrm{CDCl}_{3}\right): \delta=152.73,144.33,139.56,136.97,134.55,129.23,128.58,124.02$, $123.87,28.71,24.02$.

HRMS (ESI): m/z calcd for $\mathrm{C}_{19} \mathrm{H}_{23}{ }^{35} \mathrm{ClN}_{2}: 314.1550$, found: 314.1365 .

2,4,6-tris-(4-chloro-phenyl)-[1,3,5]-triazine (4b) Compound 4b was obtained following 
the procedure 2 catalyzed by catalyst IV and isolated as a white solid in $35 \%$ yield. Known compound. ${ }^{3}$

Mp: $327-328{ }^{\circ} \mathrm{C}\left(\right.$ lit. $\left.^{3} 330{ }^{\circ} \mathrm{C}\right)$.

${ }^{1} \mathrm{H}$ NMR $\left(\mathrm{C}_{6} \mathrm{D}_{6}\right): \delta=8.47-8.49(6 \mathrm{H}), 7.32-7.34(6 \mathrm{H})$.

HRMS (ESI): $\mathrm{m} / \mathrm{z}$ calcd for $\mathrm{C}_{21} \mathrm{H}_{15}{ }^{35} \mathrm{Cl}_{3} \mathrm{~N}_{3}$ : 414.0332, found: 414.0323 .

$\underline{\text { N'-(4-flurophenyl)-2-phenylacetamidine (3cb) }}$ Compound $3 \mathrm{cb}$ was obtained following the above procedure catalyzed by catalysts I-IV and isolated as a white solid in a $65,62,55$ and $45 \%$ yields, respectively.

Mp: $103-104{ }^{\circ} \mathrm{C}$.

$\operatorname{IR}\left(\mathrm{cm}^{-1}\right) v=3471(\mathrm{~N}-\mathrm{H}), 1617(\mathrm{C}=\mathrm{N})$.

$\mathrm{H}^{1} \mathrm{NMR}\left(\mathrm{CDCl}_{3}\right): \delta 7.32(5 \mathrm{H}), 6.97(2 \mathrm{H}), 6.83(2 \mathrm{H}), 4.45(2 \mathrm{H}), 3.62(2 \mathrm{H})$.

$\mathrm{C}^{13} \mathrm{NMR}\left(\mathrm{CDCl}_{3}\right): \delta 160.57,158.20,145.70,136.69,129.43,127.70,123.38,123.30$, $116.57,116.35,110.19,38.49$.

HRMS (ESI): m/z calcd for $\mathrm{C}_{14} \mathrm{H}_{13} \mathrm{FN}_{2}$ : 228.1063, found: 228.1057.

2,4,6-triphenyl-[1,3,5]-triazine (4a) Compound 4a was obtained following the procedure 2 catalyzed by catalyst 4 and isolated as a white solid in 30 or $60 \%$ yields respectively. Known compound. ${ }^{4}$

Mp: $236-237^{\circ} \mathrm{C}$ (lit. $\left.{ }^{4} 235-237^{\circ} \mathrm{C}\right)$;

${ }^{1} \mathrm{HNMR}\left(\mathrm{CDCl}_{3}\right): \delta 8.80(6 \mathrm{H}), 7.60(9 \mathrm{H})$.

${ }^{13} \mathrm{CNMR}\left(\mathrm{CDCl}_{3}\right): \delta 172.09,136.68,132.99,129.43,129.11$. 
HRMS (ESI): $\mathrm{m} / \mathrm{z}$ calcd for $\mathrm{C}_{21} \mathrm{H}_{18} \mathrm{~N}_{3}: 312.1501$, found: 312.1496 .

Piperidinobenzamidine (3ad) Compound 3ad was obtained following the procedure 1 catalyzed by catalysts I-III and isolated as white solid in 55, 58 and $45 \%$ yields, respectively. Known compound. ${ }^{5}$

Mp: $115-116^{\circ} \mathrm{C}$.

${ }^{1} \mathrm{H} N M R\left(\mathrm{CDCl}_{3}\right): \delta=7.35-7.56(5 \mathrm{H}), 5.86(1 \mathrm{H}$, broad $), 3.32-3.52(4 \mathrm{H}), 1.18-2.00(6 \mathrm{H})$.

HRMS (ESI): m/z calcd for $\mathrm{C}_{12} \mathrm{H}_{16} \mathrm{~N}_{2}: 188.1313$, found: 188.1301 .

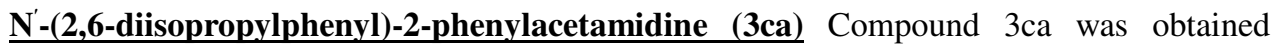
following the procedure 1 catalyzed by catalyst $\mathbf{I}$ and isolated as a white solid in a 30 or $75 \%$ yield.

Mp: $203-205^{\circ} \mathrm{C}$.

$\operatorname{IR}\left(\mathrm{cm}^{-1}\right) v=3433(\mathrm{~N}-\mathrm{H}), 1635(\mathrm{C}=\mathrm{N})$.

${ }^{1} \mathrm{H}$ NMR $\left(\mathrm{CDCl}_{3}\right): \delta=7.38-7.40(5 \mathrm{H}), 7.06-7.12(3 \mathrm{H}), 4.15(2 \mathrm{H}), 3.77(2 \mathrm{H}), 2.96-3.13$ (2H), 1.11-1.19 (12H).

${ }^{13} \mathrm{C} \mathrm{NMR}\left(\mathrm{CDCl}_{3}\right): \delta=155.73,144.05,139.95,137.15,129.56,129.37,127.69,123.86$, $123.74,43.65,28.44,24.21,24.08$.

HRMS (ESI): m/z calcd for $\mathrm{C}_{20} \mathrm{H}_{26} \mathrm{~N}_{2}$ : 294.2096, found: 294.2091.

$\underline{\text { N'-(2,6-diisopropylphenyl)-4-methoxybenamidine (3da) Compound 3da was obtained }}$ following the procedure 1 catalyzed by catalyst $\mathbf{I}$ and isolated as a white solid in a $92 \%$ 
yield.

Mp: $146-147^{\circ} \mathrm{C}$.

IR $\left(\mathrm{cm}^{-1}\right) v=3498(\mathrm{~N}-\mathrm{H}), 1629(\mathrm{C}=\mathrm{N})$.

${ }^{1} \mathrm{HNMR}\left(\mathrm{CDCl}_{3}\right): \delta=7.87-7.90(2 \mathrm{H}), 7.15-7.18(2 \mathrm{H}), 7.08-7.10(1 \mathrm{H}), 6.97-6.99(2 \mathrm{H}), 4.55$

$(2 \mathrm{H}), 3.87(3 \mathrm{H}), 2.99-3.10(2 \mathrm{H}), 1.18-1.20(12 \mathrm{H})$.

${ }^{13} \mathrm{C} \mathrm{NMR}\left(\mathrm{CDCl}_{3}\right): \delta=161.64,153.04,144.57,139.61,128.46,128.42,123.55,123.50$,

$114.05,55.68,28.42,23.95,23.81$.

HRMS (ESI): $\mathrm{m} / \mathrm{z}$ calcd for $\mathrm{C}_{20} \mathrm{H}_{25} \mathrm{~N}_{2} \mathrm{O}: 310.2045$, found: 310.2041 .

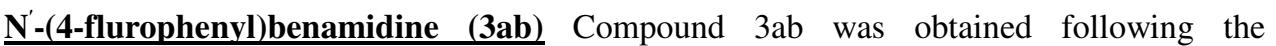
procedure 1 catalyzed by catalyst I and isolated as a white solid in a 97\% yield.

Mp: $126-128{ }^{\circ} \mathrm{C}$.

$\operatorname{IR}\left(\mathrm{cm}^{-1}\right) v=3472(\mathrm{~N}-\mathrm{H}), 1616(\mathrm{C}=\mathrm{N})$.

${ }^{1} \mathrm{HNMR}\left(\mathrm{CDCl}_{3}\right): \delta=7.83(2 \mathrm{H}), 7.45(3 \mathrm{H}), 7.03(2 \mathrm{H}), 6.92(2 \mathrm{H}), 4.89(2 \mathrm{H})$.

${ }^{13} \mathrm{C} \mathrm{NMR}\left(\mathrm{CDCl}_{3}\right): \delta=161.04,157.84,155.72,146.06,136.06,131.07,128.97,127.18$, $123.27,123.17,116.71,116.42$

HRMS (ESI): $\mathrm{m} / \mathrm{z}$ calcd for $\mathrm{C}_{13} \mathrm{H}_{11} \mathrm{FN}_{2}: 214.0906$, found: 214.0890 .

N'-(4-flurophenyl)-4-methoxylbenamidine (3db) Compound $3 \mathrm{db}$ was obtained following

Deleted: $\underline{\text { 3eb }}$

Deleted: $3 \mathrm{eb}$ the procedure 1 catalyzed by catalyst $\mathbf{I}$ and isolated as a white solid in a $98 \%$ yield.

Mp: $147-149^{\circ} \mathrm{C}$.

IR $\left(\mathrm{cm}^{-1}\right) v=3498(\mathrm{~N}-\mathrm{H}), 1630(\mathrm{C}=\mathrm{N})$.

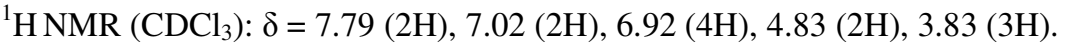


${ }^{13} \mathrm{C} \mathrm{NMR}\left(\mathrm{CDCl}_{3}\right): \delta=161.95,160.97,157.78,155.34,146.23,128.72,123.39,116.67$,

$116.37,114.17,55.81$.

HRMS (ESI): $\mathrm{m} / \mathrm{z}$ calcd for $\mathrm{C}_{14} \mathrm{H}_{13} \mathrm{FN}_{2} \mathrm{O}: 244.1012$, found: 244.1007 .

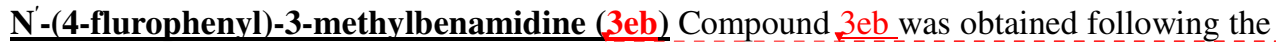

Deleted: $\underline{3 f b}$

procedure 1 catalyzed by catalyst $\mathbf{I}$ and isolated as a white solid in a $95 \%$ yield.

Mp: $118-119^{\circ} \mathrm{C}$.

$\operatorname{IR}\left(\mathrm{cm}^{-1}\right) v=3447(\mathrm{~N}-\mathrm{H}), 1626(\mathrm{C}=\mathrm{N})$.

${ }^{1} \mathrm{H}$ NMR $\left(\mathrm{CDCl}_{3}\right): \delta=7.73(1 \mathrm{H}), 7.62-7.63(1 \mathrm{H}), 7.33(2 \mathrm{H}), 7.04(2 \mathrm{H}), 6.95(2 \mathrm{H}), 4.86$ $(2 \mathrm{H}), 2.41(3 \mathrm{H})$.

${ }^{13} \mathrm{C} \mathrm{NMR}\left(\mathrm{CDCl}_{3}\right): \delta=160.88,157.75,155.67,146.05,138.69,135.84,131.69,128.69$, $127.82,123.85,123.10,116.57,116.28,21.64$

HRMS (ESI): m/z calcd for $\mathrm{C}_{14} \mathrm{H}_{13} \mathrm{FN}_{2}$ : 228.1063, found: 228.1056.

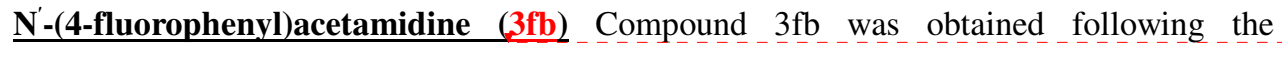
procedure 1 catalyzed by catalyst $\mathbf{I}$ and isolated as a white solid in a 45 and $75 \%$ yield.

Mp: $145-147^{\circ} \mathrm{C}$.

$\operatorname{IR}\left(\mathrm{cm}^{-1}\right) v=3450(\mathrm{~N}-\mathrm{H}), 1628(\mathrm{C}=\mathrm{N})$.

${ }^{1} \mathrm{HNMR}\left(\mathrm{CDCl}_{3}\right): \delta=6.90(2 \mathrm{H}), 6.76(2 \mathrm{H}), 4.33(2 \mathrm{H}), 3.56(3 \mathrm{H})$.

${ }^{13} \mathrm{C} \mathrm{NMR}\left(\mathrm{CDCl}_{3}\right): \delta=160.46,158.09,123.40,123.33,116.45,116.25,38.49$

HRMS (ESI): m/z calcd for $\mathrm{C}_{8} \mathrm{H}_{9} \mathrm{FN}_{2}$ : 152.0750 , found: 152.0756 .

2,4,6-tri-pyridin-4-yl-[1,3,5]triazine (4g) Compound 4g was obtained following the 
procedure 2 catalyzed by catalyst $\mathbf{I}$ and isolated as a white solid in a $95 \%$ yield. Known compound. $^{8}$

Mp: $372-374{ }^{\circ} \mathrm{C}$.

${ }^{1} \mathrm{H}$ NMR $\left(\mathrm{C}_{6} \mathrm{D}_{6}\right): \delta=8.83-8.84(6 \mathrm{H}), 8.46-8.47(6 \mathrm{H})$.

HRMS (ESI): m/z calcd for $\mathrm{C}_{18} \mathrm{H}_{15} \mathrm{~N}_{6}: 315.1358$, found: 315.1350 .

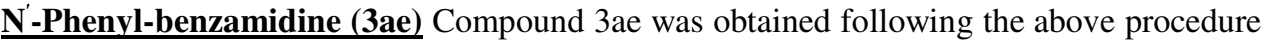
1 catalyzed by catalyst I and isolated as a white solid in a $94 \%$ yield. Known compound. ${ }^{6}$ Mp: $114-115^{\circ} \mathrm{C}\left(\operatorname{lit}^{6} 115^{\circ} \mathrm{C}\right)$.

${ }^{1} \mathrm{H} \mathrm{NMR}\left(\mathrm{CDCl}_{3}\right): \delta=7.92(2 \mathrm{H}), 7.40(5 \mathrm{H}), 7.02(3 \mathrm{H}), 4.86(2 \mathrm{H})$.

$\underline{\mathbf{N}^{\prime}-(2,6-d i m e t h y l p h e n y l) b e n a m i d i n e ~(3 a f)}$ Compound 3af was obtained following the procedure 1 catalyzed by catalyst $\mathbf{I}$ and isolated as a white solid in a $91 \%$ yield. Known compound. ${ }^{7}$

Mp: $104-106{ }^{\circ} \mathrm{C}$ (lit. ${ }^{7}$ 102-105).

$\operatorname{IR}\left(\mathrm{cm}^{-1}\right) v=3432(\mathrm{~N}-\mathrm{H}), 1636(\mathrm{C}=\mathrm{N})$.

${ }^{1} \mathrm{H}$ NMR $\left(\mathrm{CDCl}_{3}\right): \delta=7.92-7.94(2 \mathrm{H}), 7.45-7.51(3 \mathrm{H}), 7.06-7.08(2 \mathrm{H}), 6.89-6.93(1 \mathrm{H})$, $4.60(2 \mathrm{H}), 2.16(6 \mathrm{H})$.

${ }^{13} \mathrm{C} \mathrm{NMR}\left(\mathrm{CDCl}_{3}\right): \delta=153.51,146.71,136.04,131.02,129.29,129.06,128.62,127.19$, $123.31,18.28$.

HRMS (ESI): $\mathrm{m} / \mathrm{z}$ calcd for $\mathrm{C}_{15} \mathrm{H}_{16} \mathrm{~N}_{2}$ : 224.1313, found: 224.1303 .

\section{Copies of ${ }^{1} \mathrm{H}$ NMR and ${ }^{13} \mathrm{C}$ NMR:}




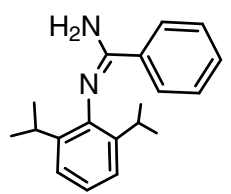

Compound 3aa ${ }^{1} \mathrm{H}$ NMR

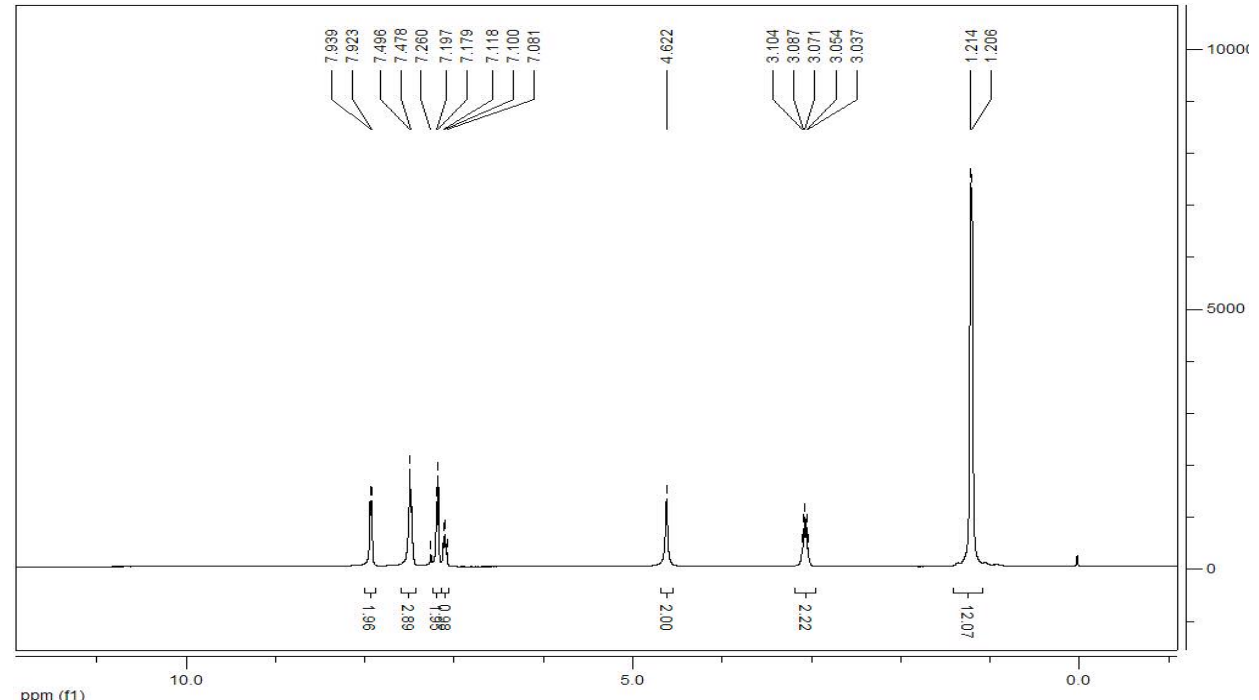

Compound 3aa ${ }^{13} \mathrm{C}$ NMR

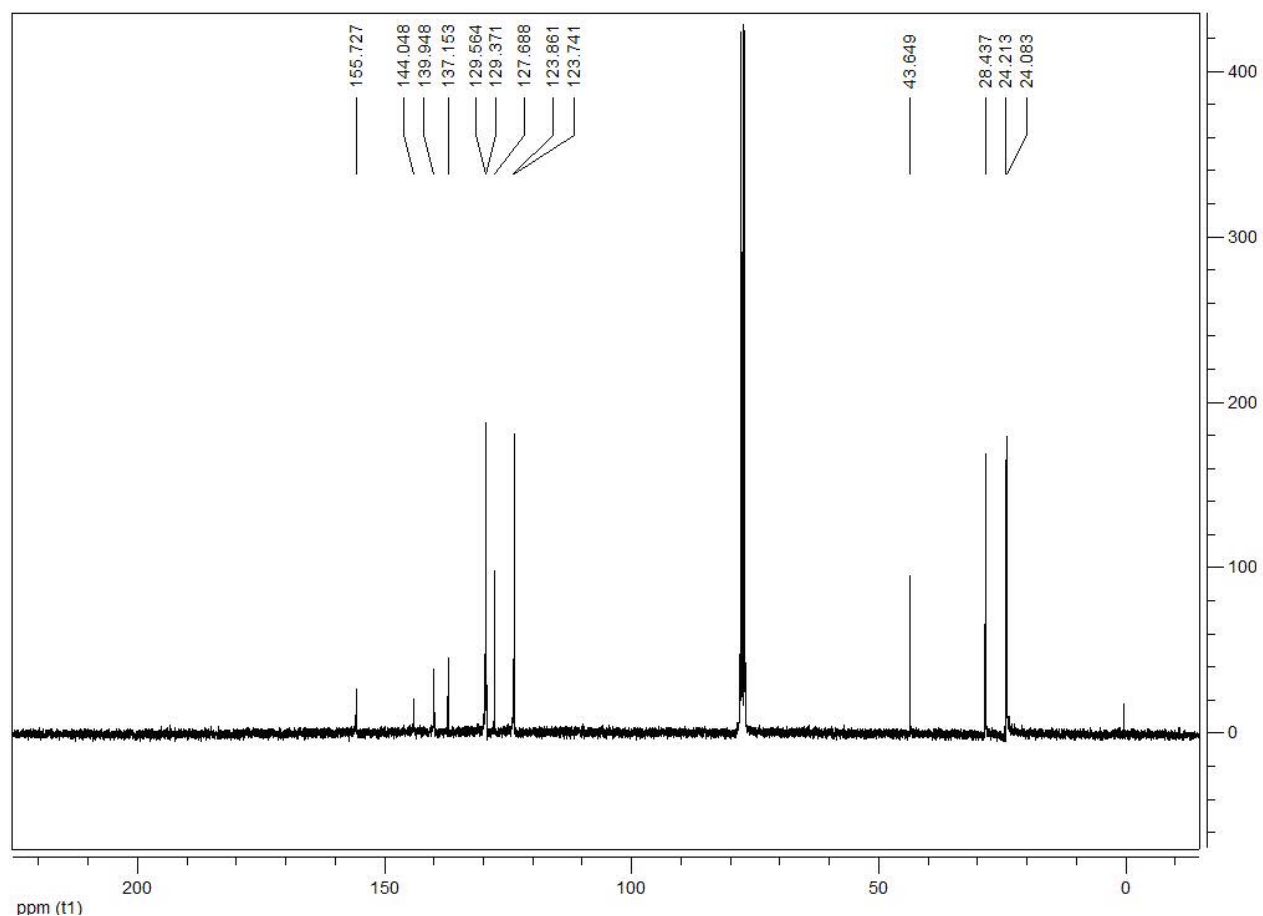




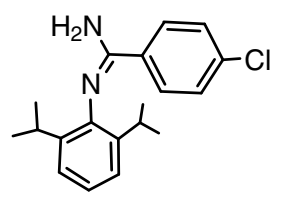

Compound 3 ba ${ }^{1} \mathrm{H}$ NMR

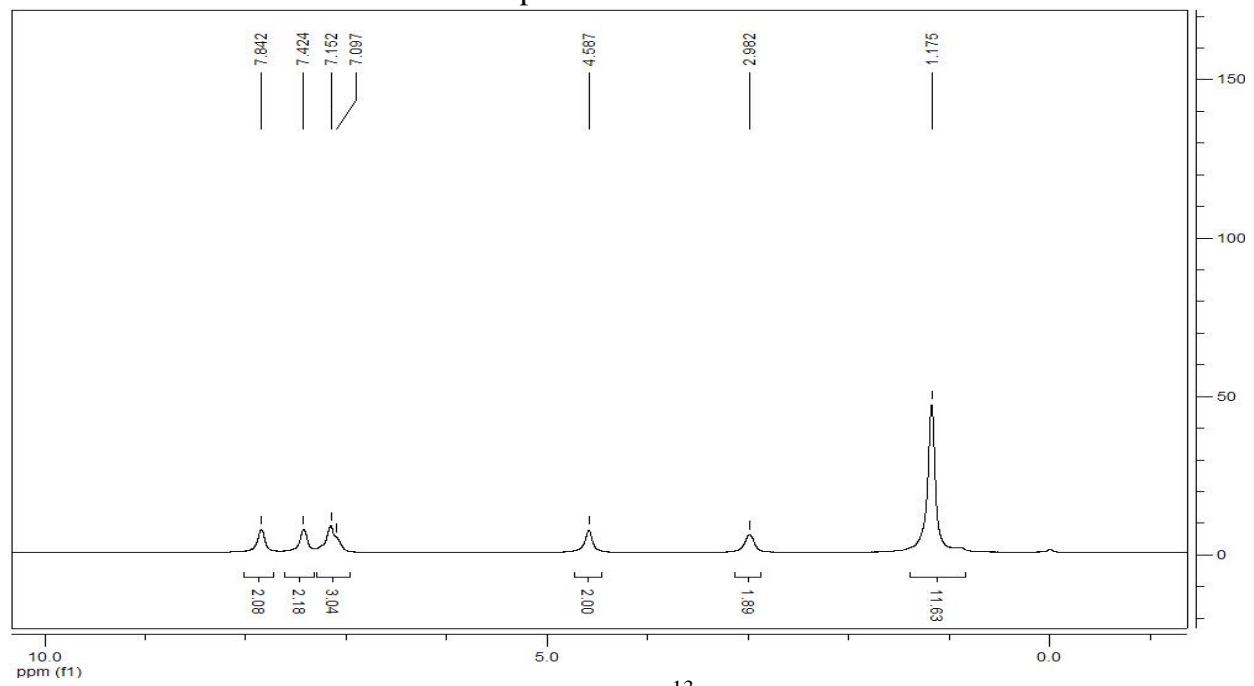

Compound 3ba ${ }^{13} \mathrm{C}$ NMR

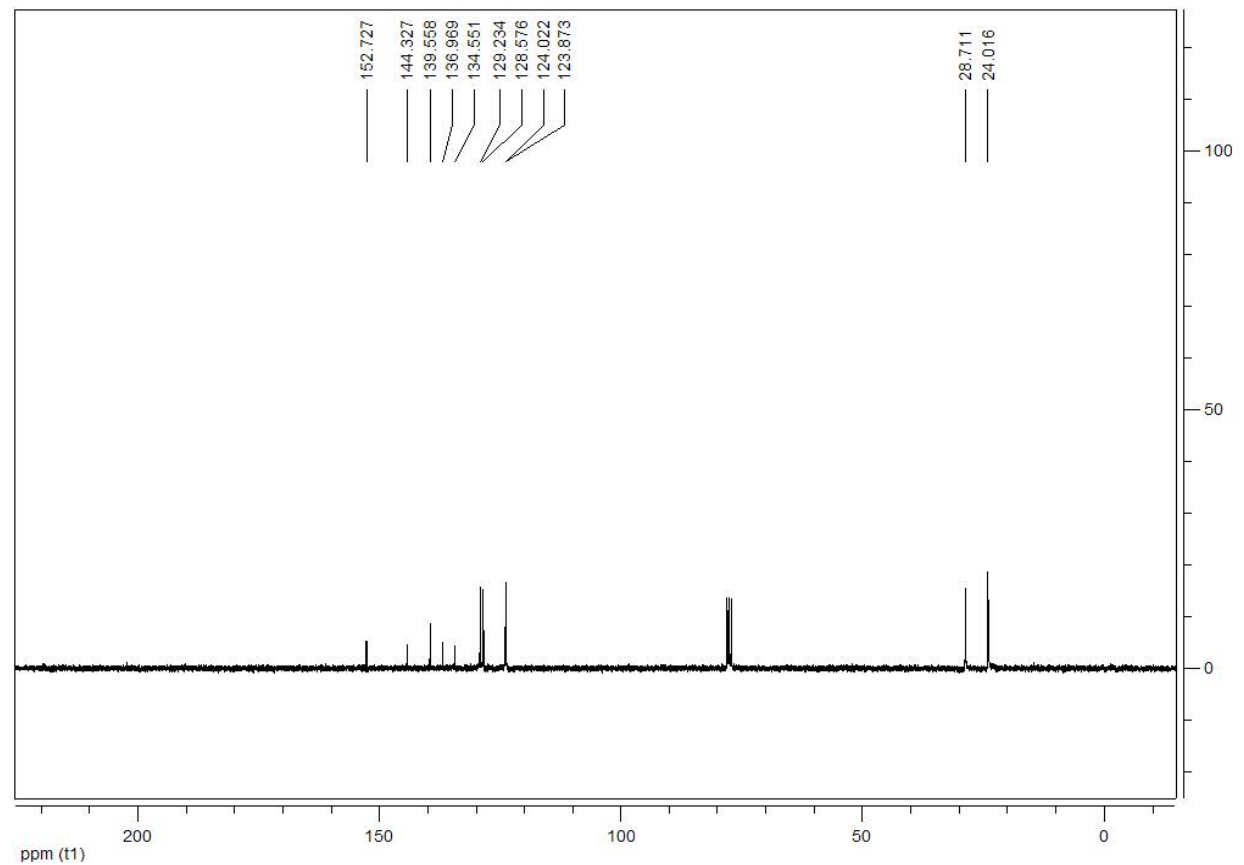




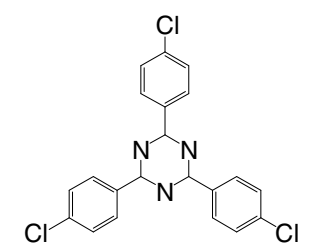

Compound $\mathbf{4 b}{ }^{1} \mathrm{H}$ NMR

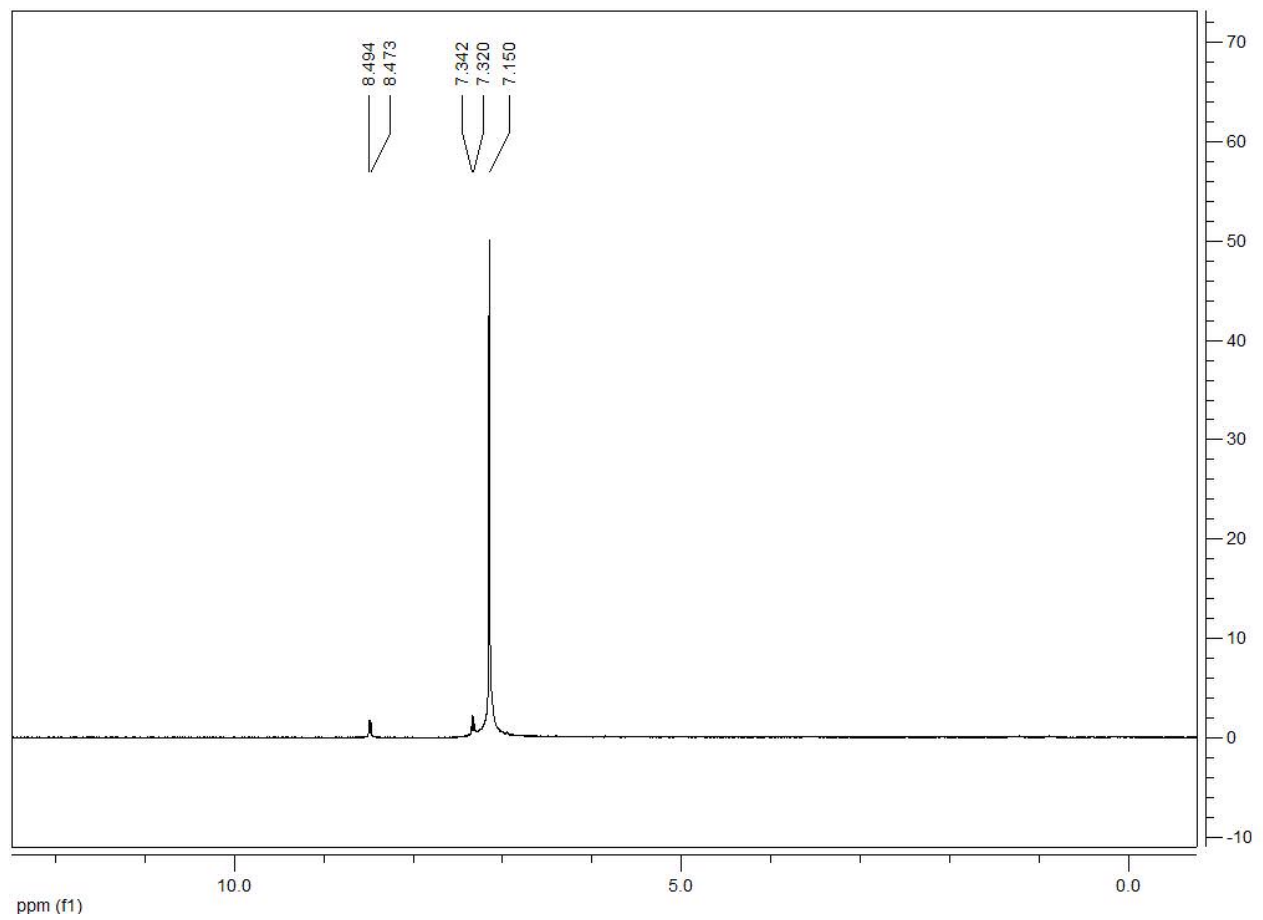




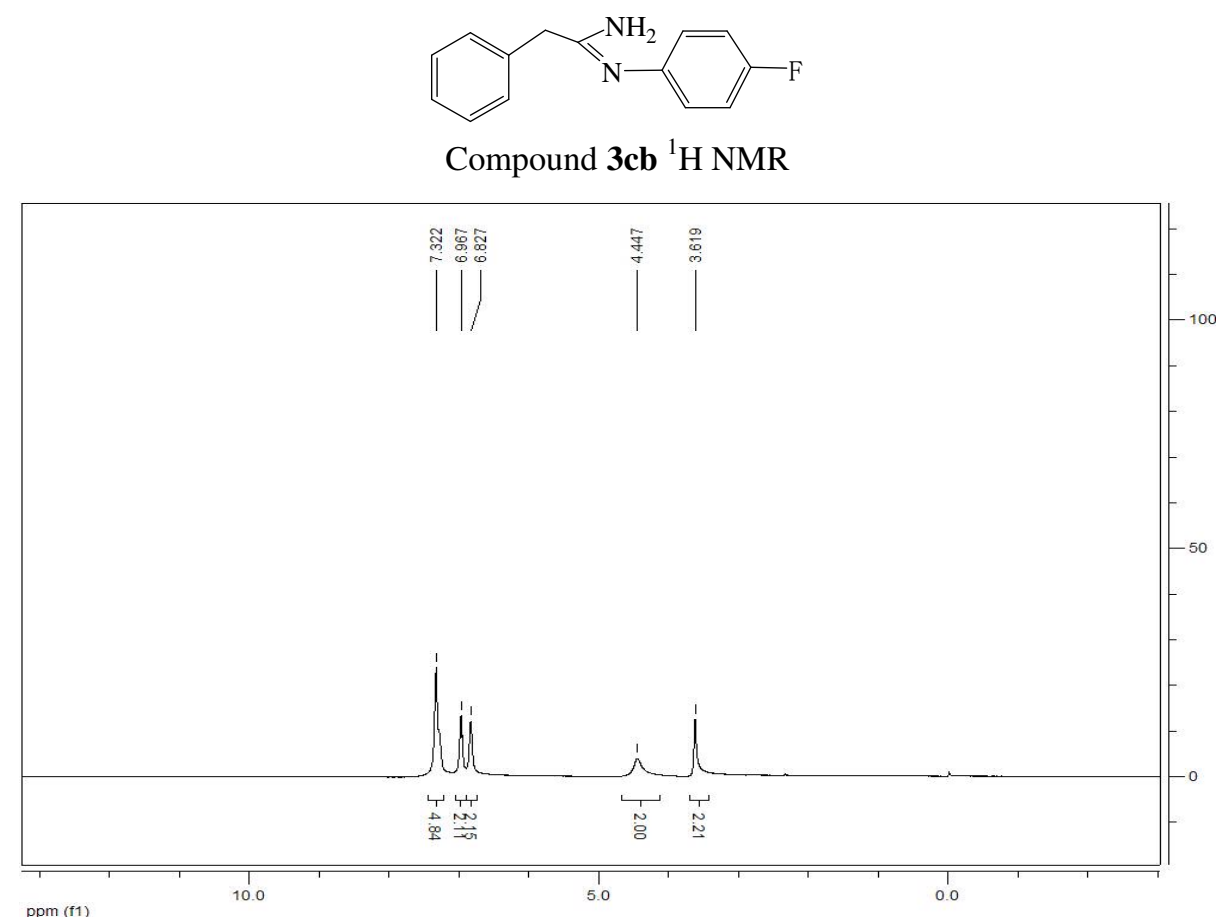

ppm (f1)

Compound 3cb ${ }^{13} \mathrm{C}$ NMR

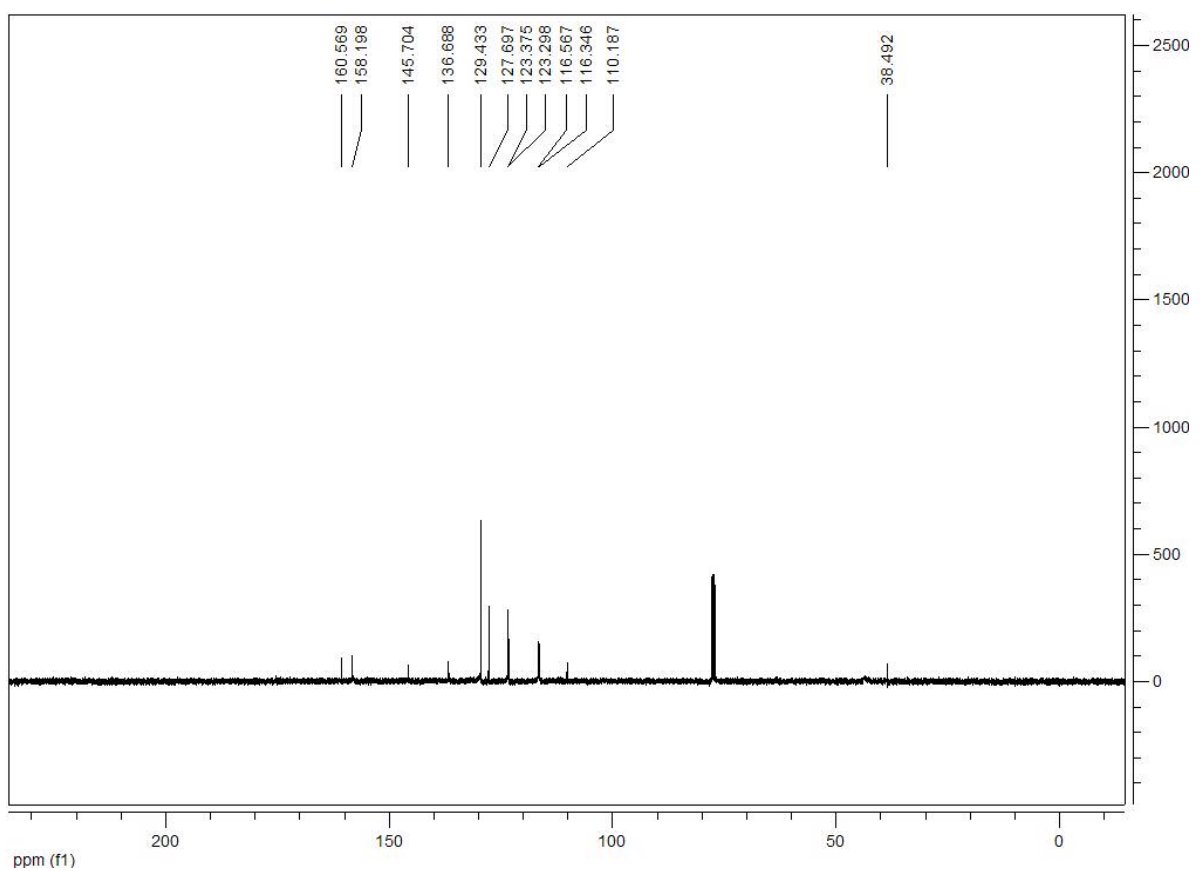




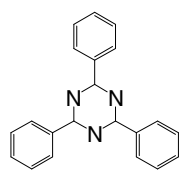

Compound $4 \mathbf{a}^{1} \mathrm{H}$ NMR

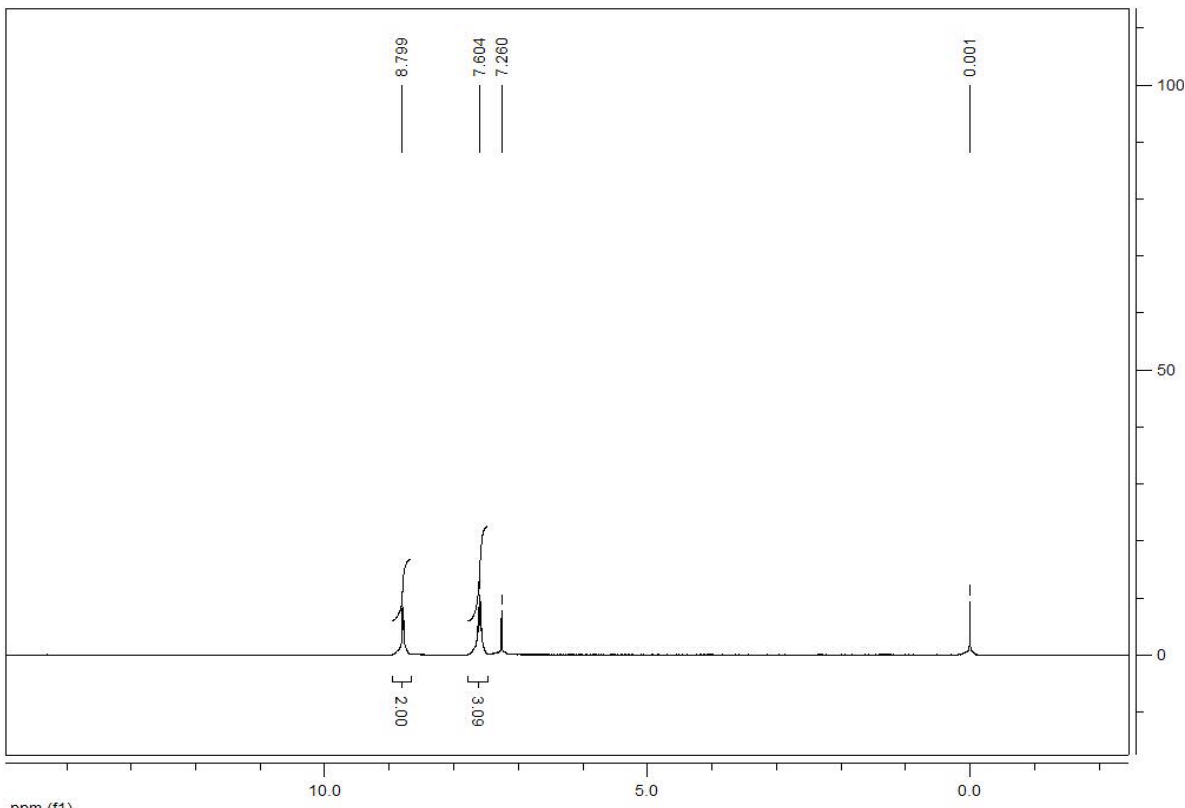

Compound $4 a^{13} \mathrm{C}$ NMR

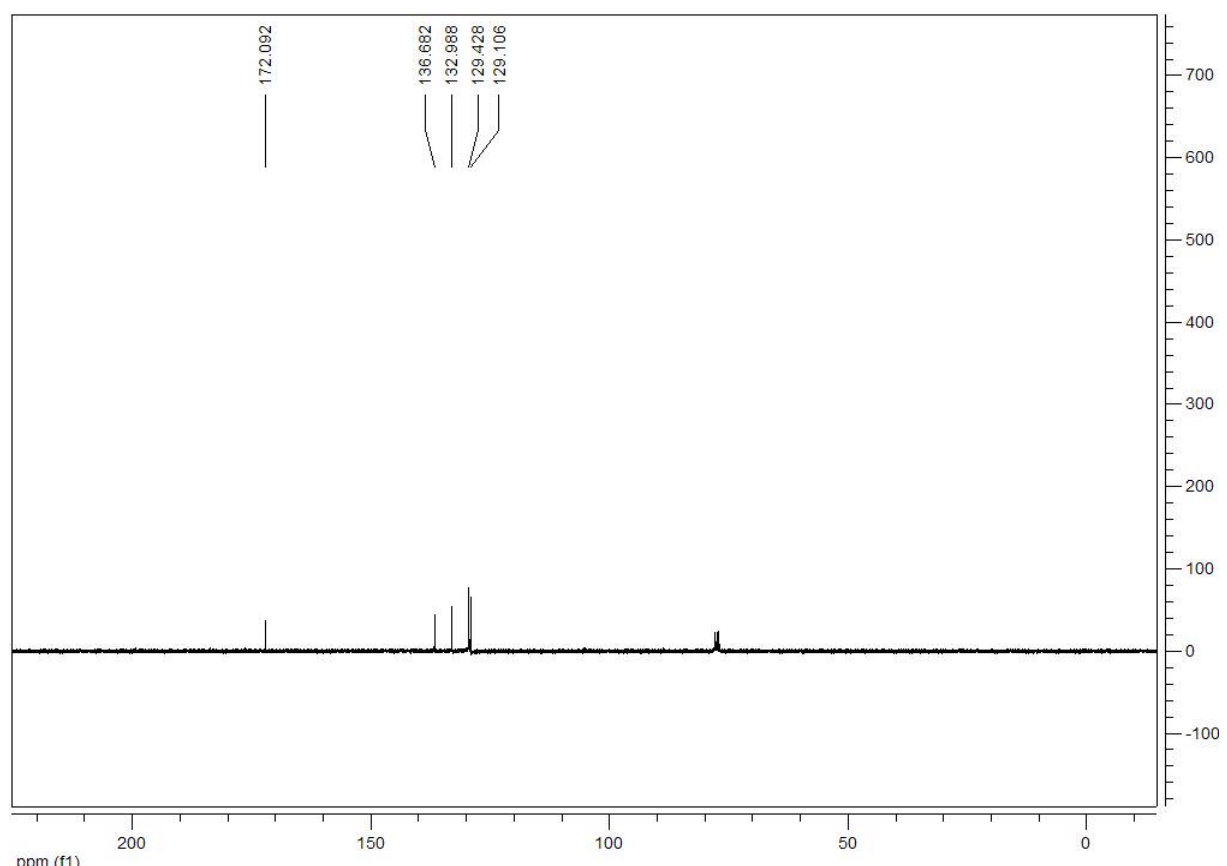

13 


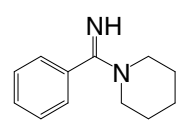

Compound 3ad ${ }^{1} \mathrm{H}$ NMR

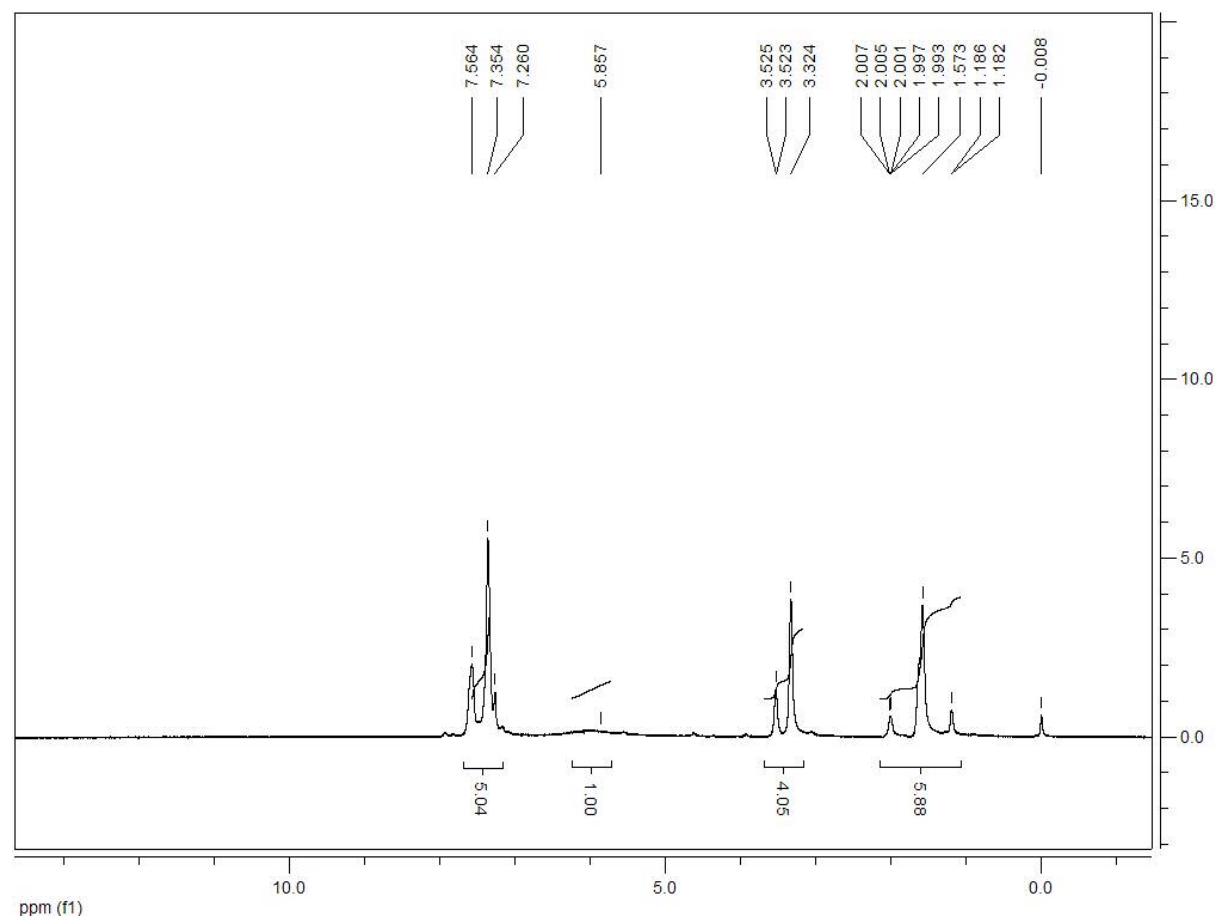




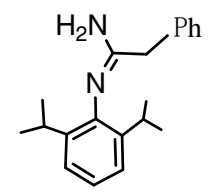

Compound 3ca ${ }^{1} \mathrm{H}$ NMR

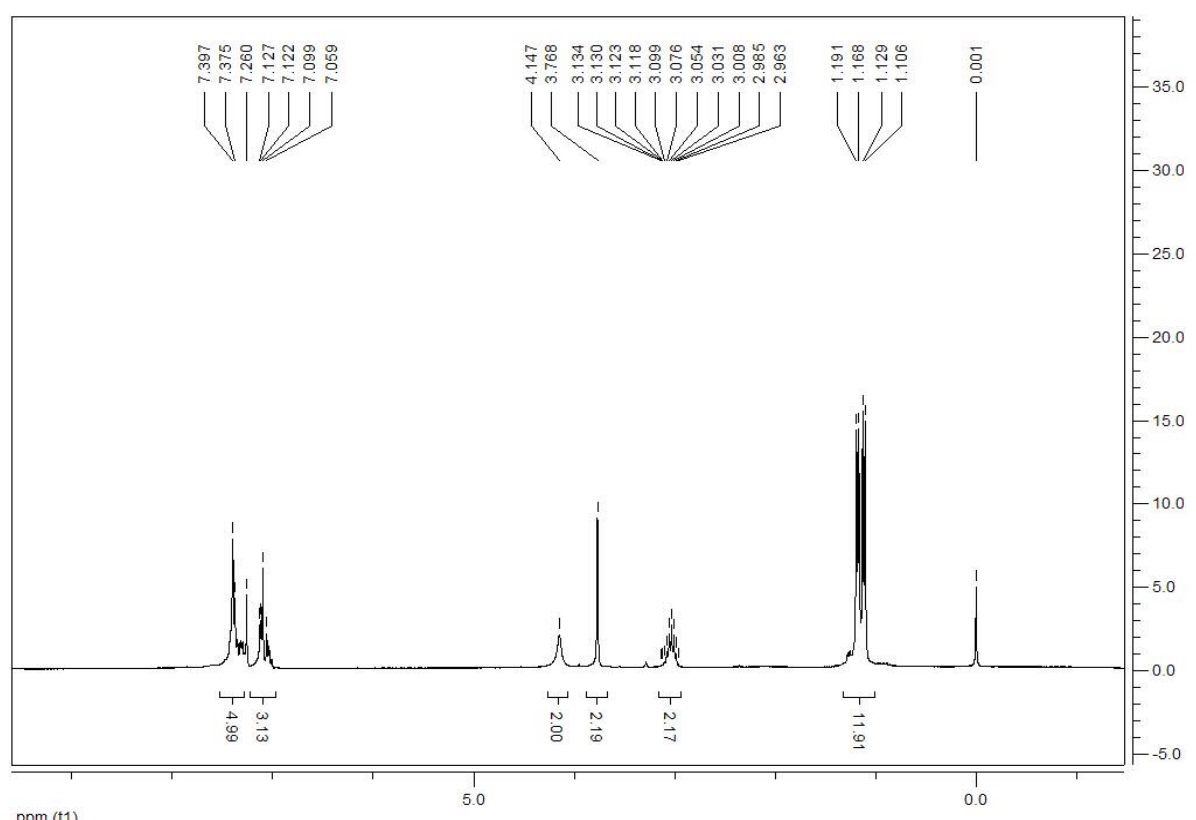

Compound 3ca ${ }^{13} \mathrm{C}$ NMR

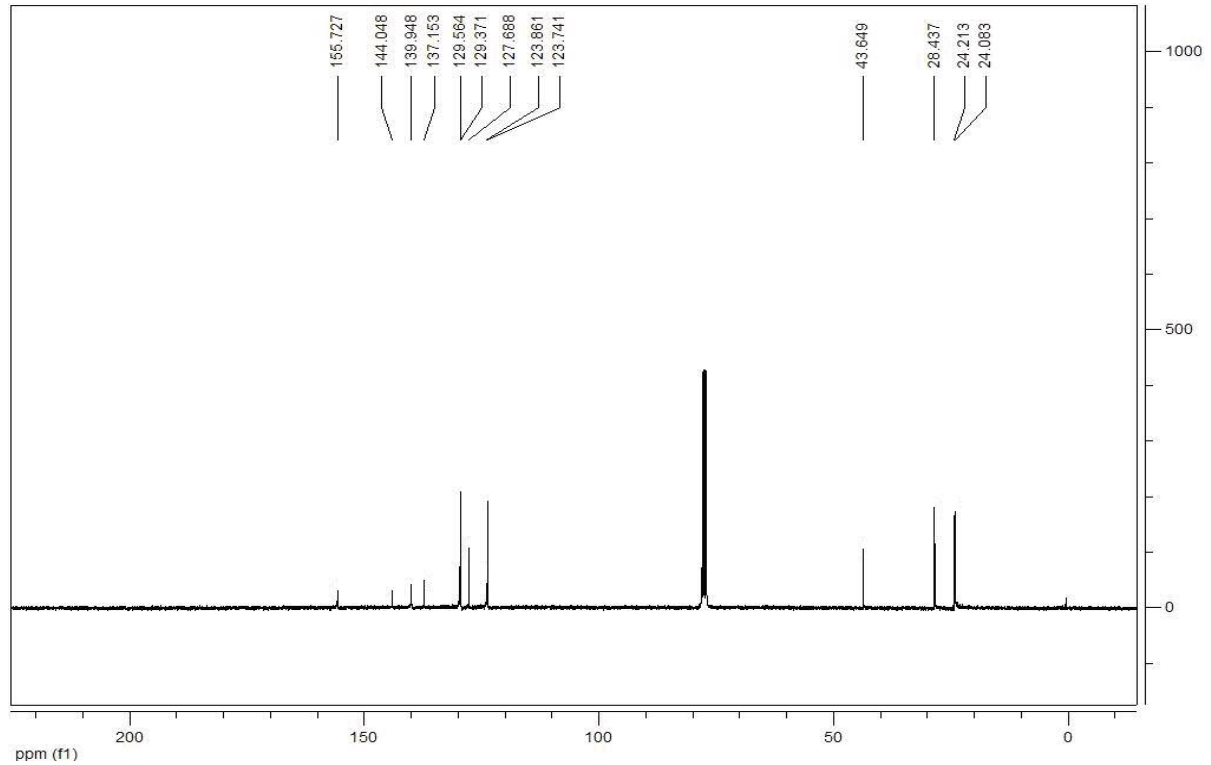




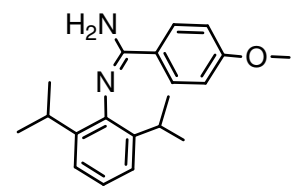

Compound 3da ${ }^{1} \mathrm{H}$ NMR

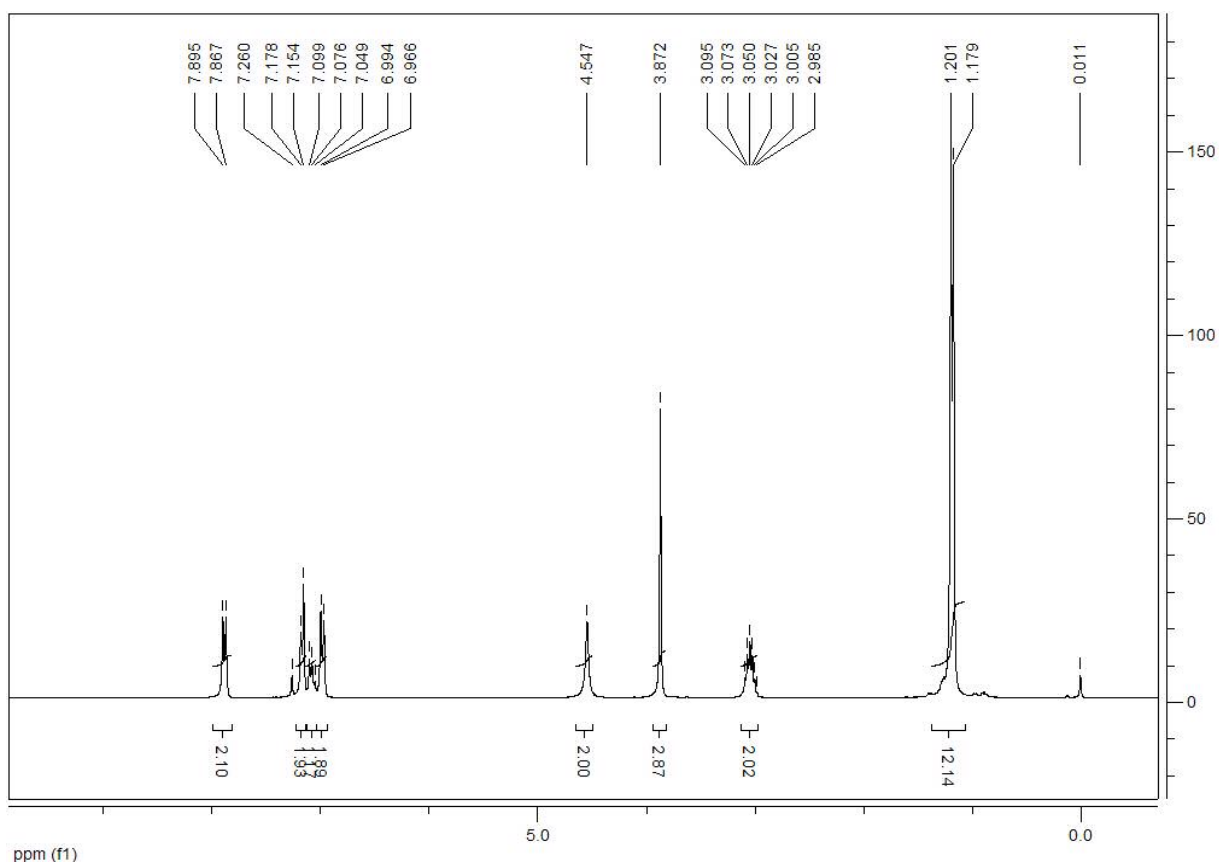

ppm (f1)

Compound 3da ${ }^{13} \mathrm{C}$ NMR

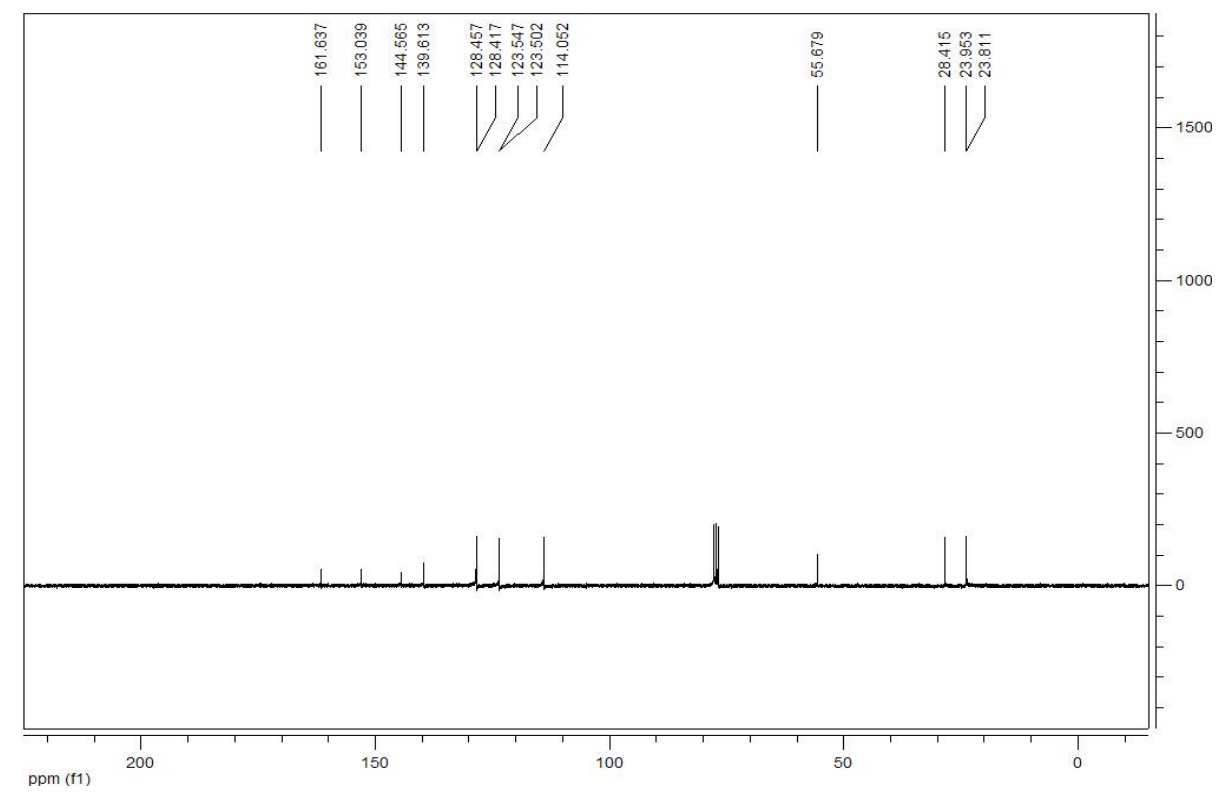

16 


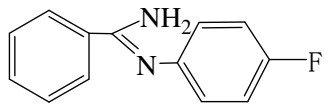

Compound 3ab ${ }^{1} \mathrm{H}$ NMR

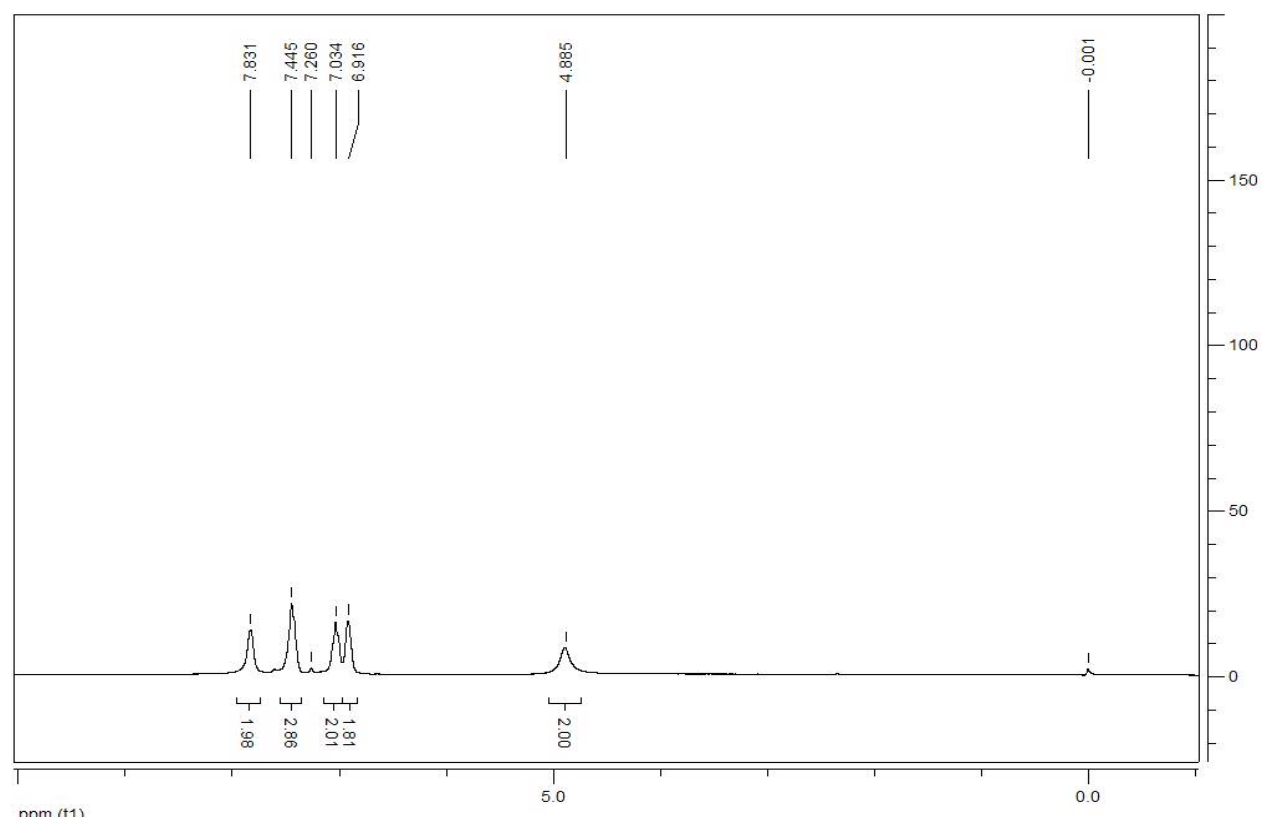

Compound 3ab ${ }^{13} \mathrm{C}$ NMR

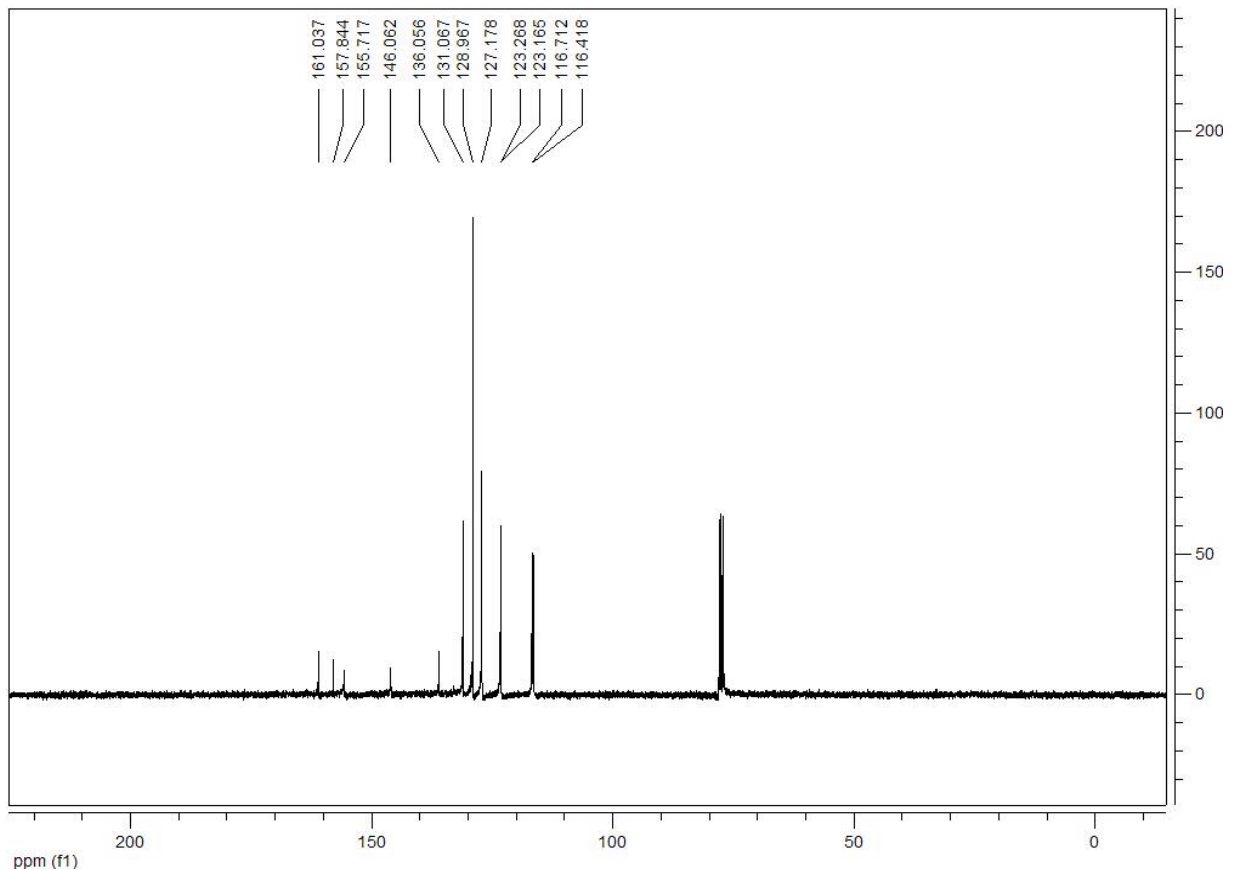

17 


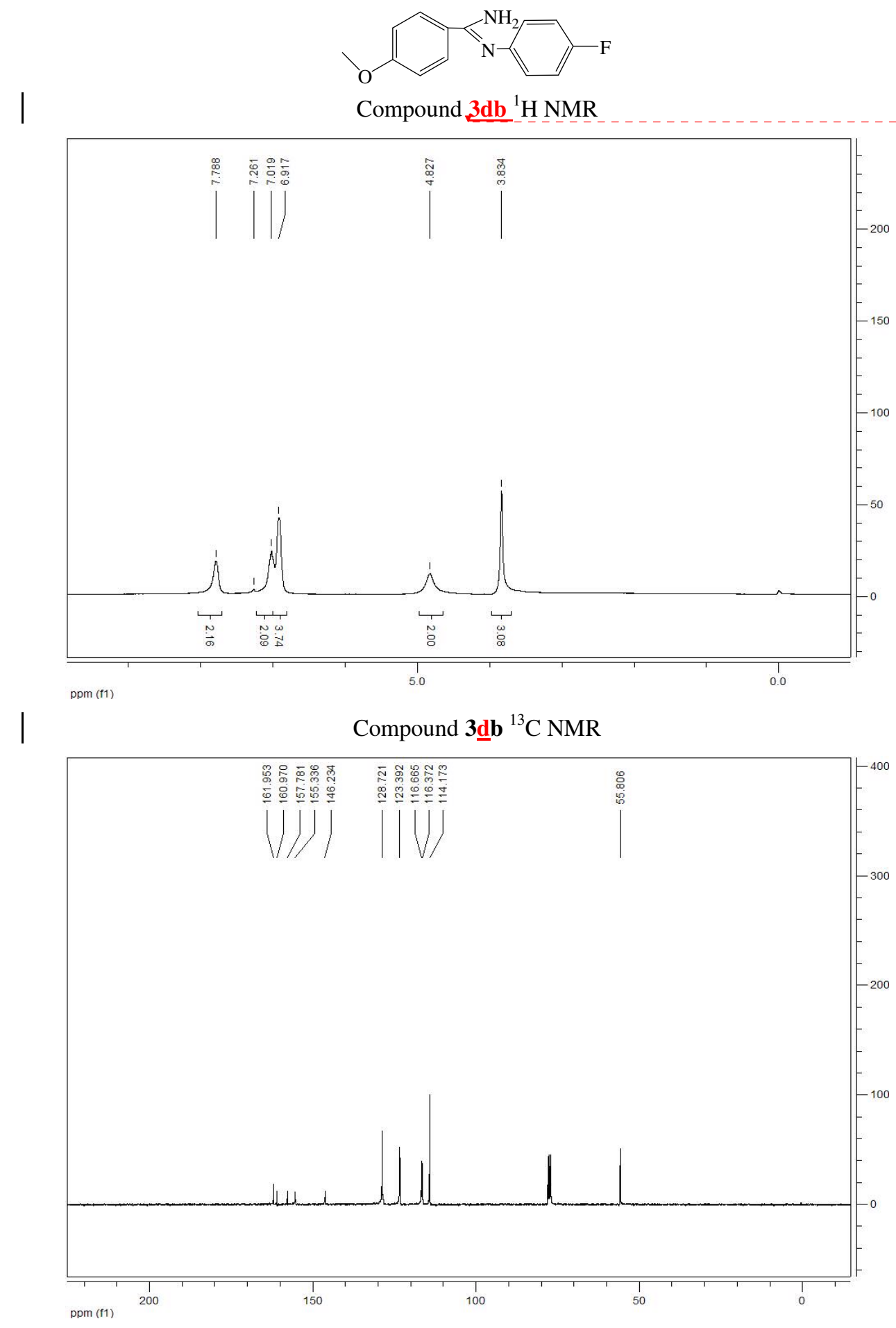




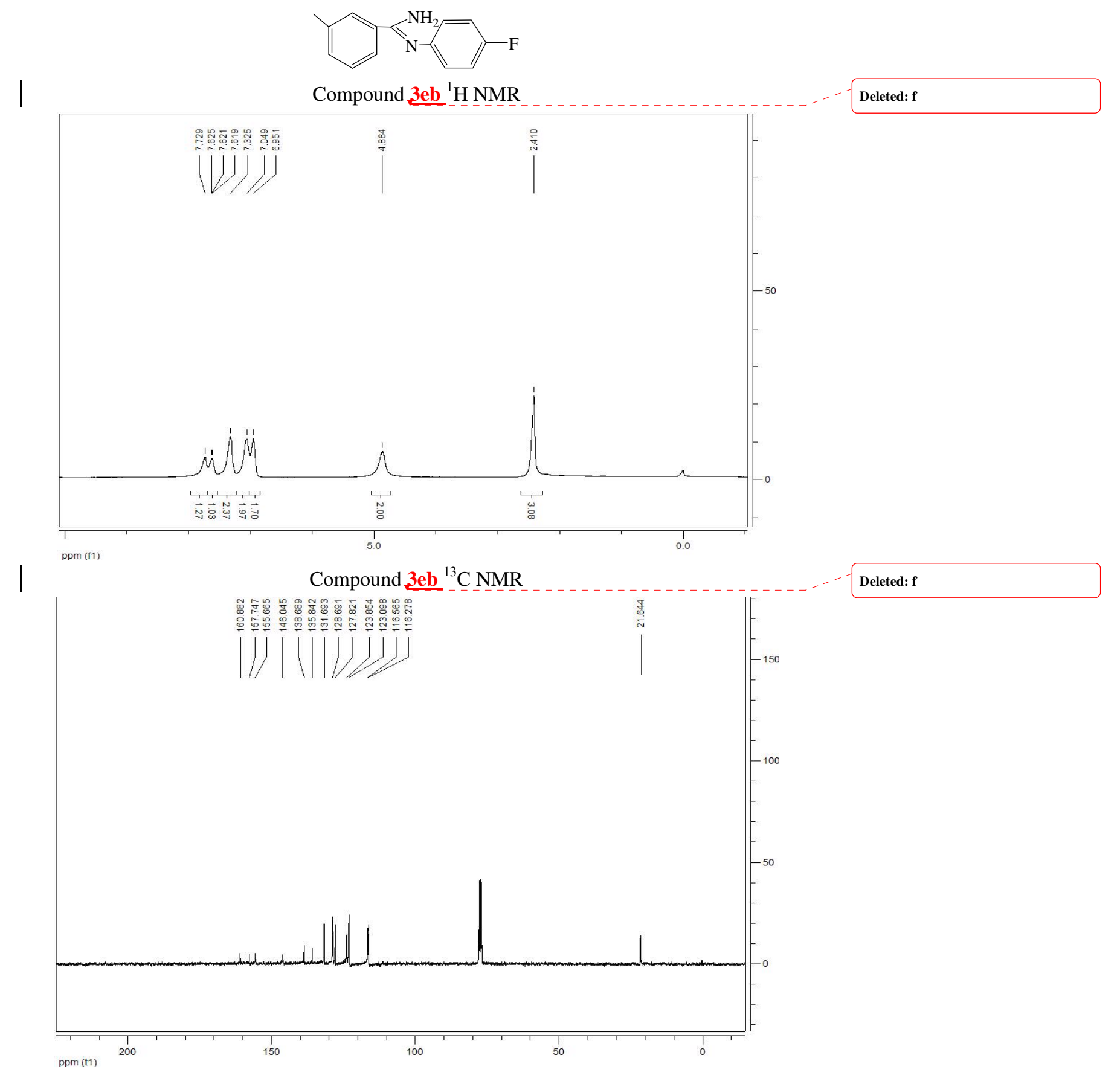



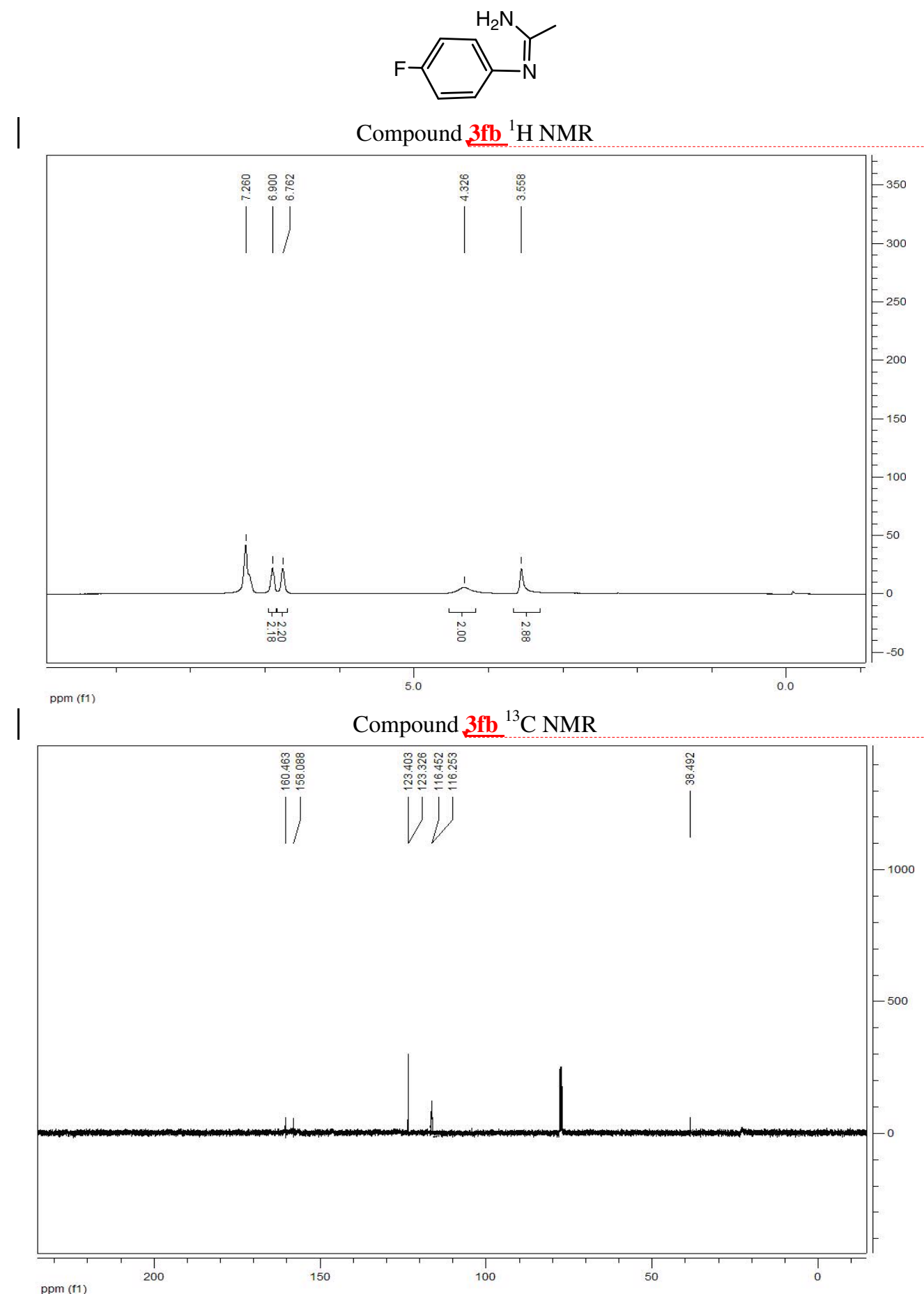

Deleted: 3gb 


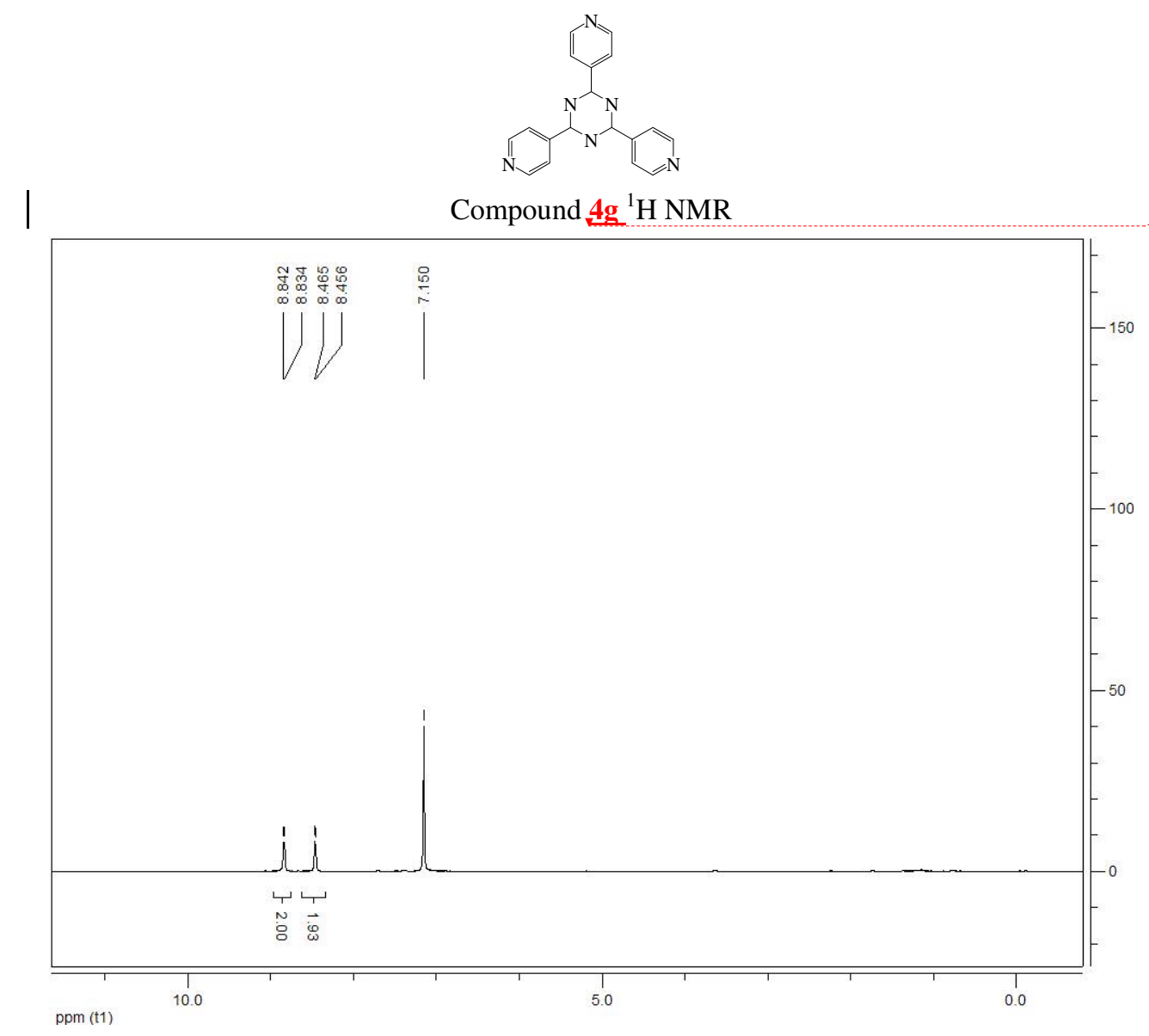




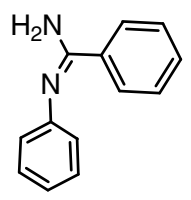

Compound 3ae ${ }^{1} \mathrm{H}$ NMR

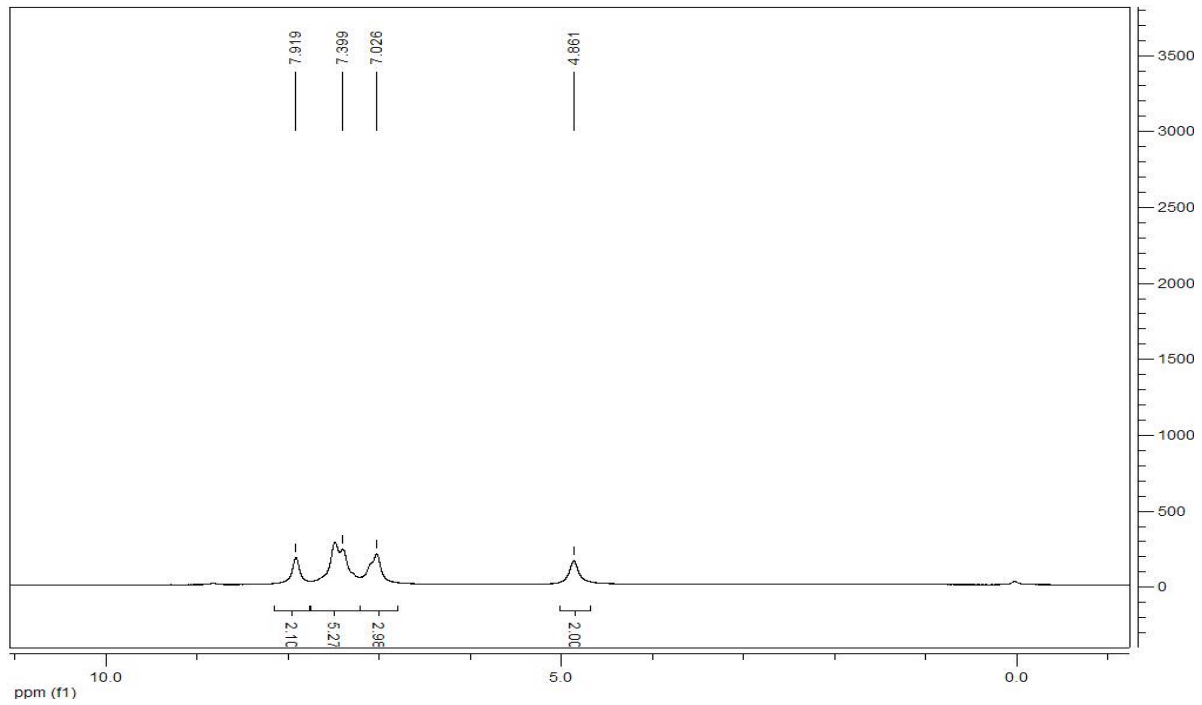




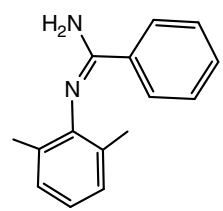

Compound 3af ${ }^{1} \mathrm{H}$ NMR

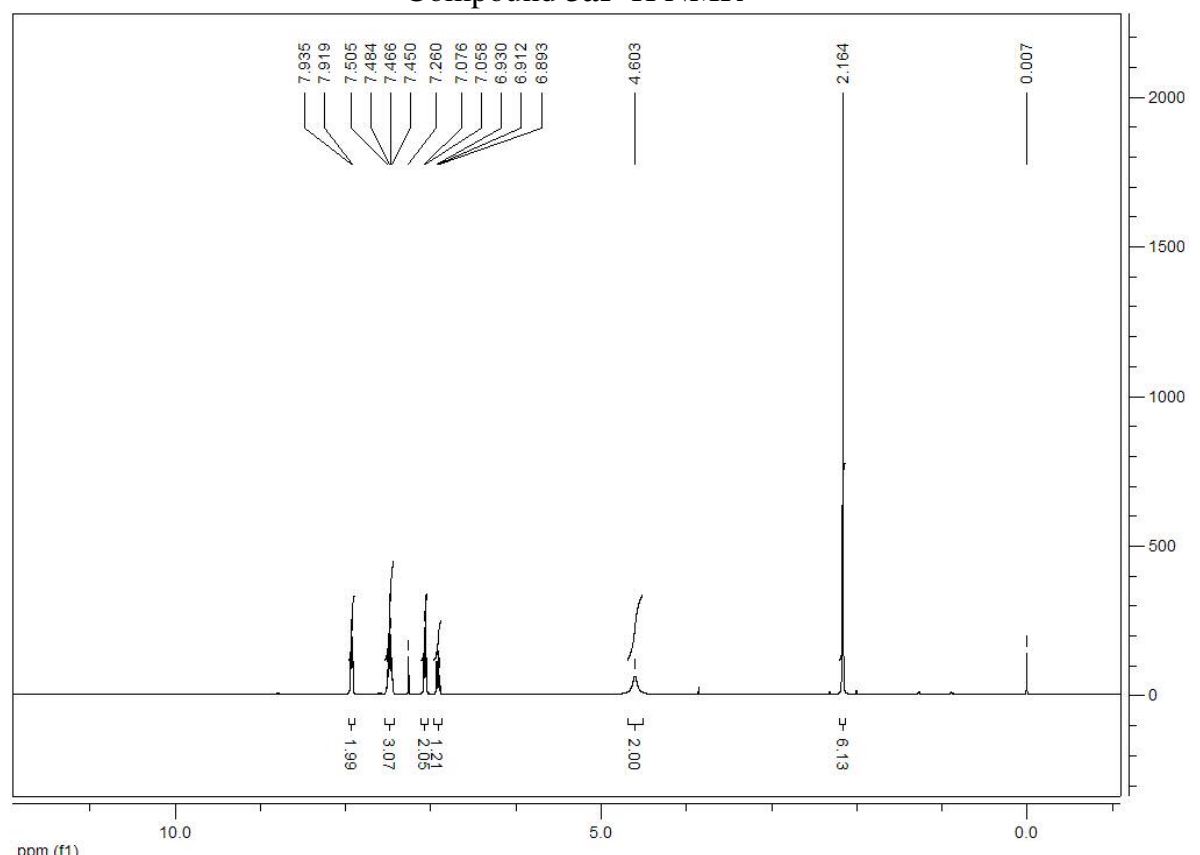

ppm (f1)

Compound 3af ${ }^{13} \mathrm{C}$ NMR

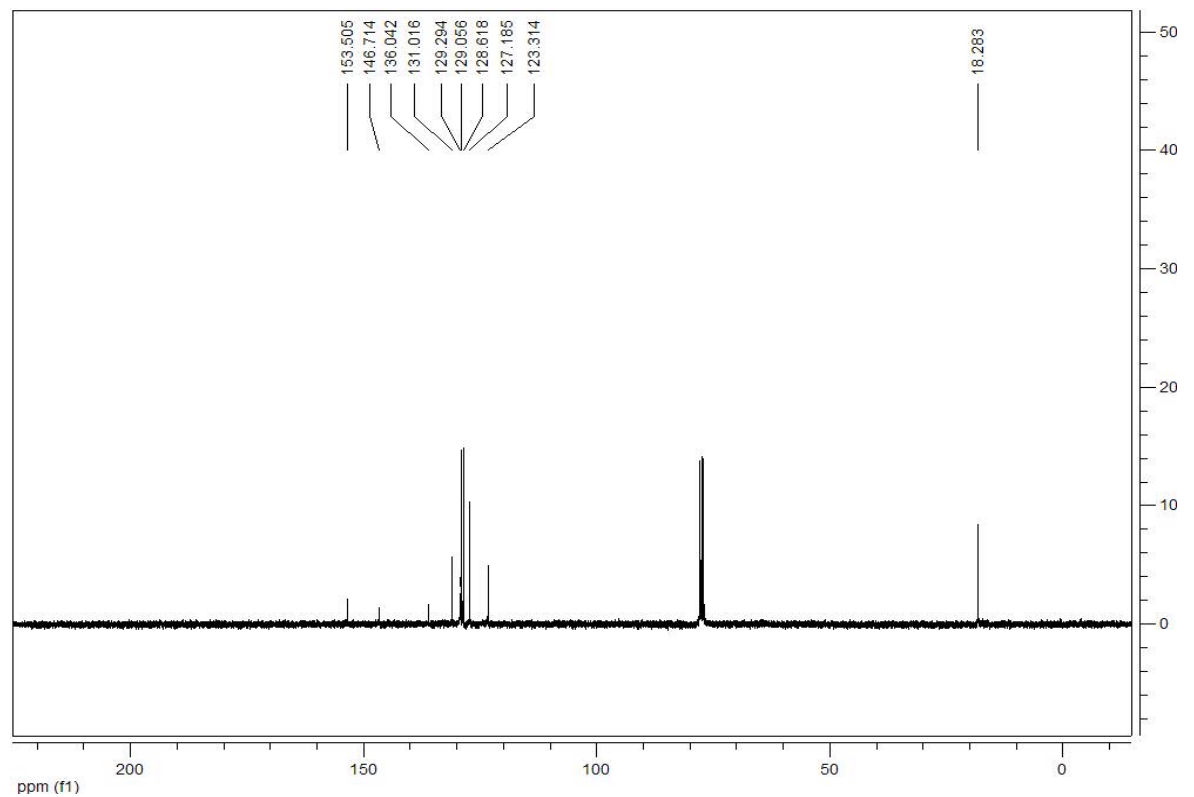




\section{Reference}

(1) Wang, J.; Cai, T.; Yao, Y.; Zhang, Y.; Shen, Q.; Dalton Trans. 2007, 5275.

(2) Zhou, S.; Wang, S.; Yang, G.; Liu, X.; Sheng, E.; Zhang, K.; Cheng, L.; Huang, Z. Polyhedron 2003, 22, 1019.

(3) LLOBERA, A.; SAA, J. M. Synthesis 1985, 1, 95.

(4) Pavlik, J. W.; Changtong, C.; Tsefrikas, V. M. J. Org. Chem. 2003, 68, 4855.

(5) Oxiey. P.; Partridge, M. W.; Short, W. F. J. Chem. Soc. 1947, 1110.

(6) Rodriguez, H.; Pavez, H.; Marquez, A.; Navarrete, P. Tetrahedron 1983, 39, 23.

(7) Gilchrist, T. L.; Christopher, J. M.; Rees, C. W. J. Chem. Soc. Perkin Trans. 1. 1979, 1871.

(8) Anderson, H. L.; Anderson, S., Sander, J. K. M. J. Chem. Soc. Perkin Trans. 1. 1995, 2231. 